\title{
Differential games of inf-sup type and Isaacs equations
}

\author{
Hidehiro Kaise And Shuenn-Jyi Sheu \\ Institute of Mathematics, Academia Sinica, \\ Nankang, Taipei 11529, Taiwan, R.O.C. \\ E-mail: kaise@math.sinica.edu.tw,sheusj@math.sinica.edu.tw
}

\begin{abstract}
Motivated by the work of Fleming [5], we shall provide the general framework to associate inf-sup type values with the Isaacs equations. We shall show that upper and lower bounds for the generators of inf-sup type are upper and lower Hamiltonian in differential games respectively. In particular, lower (resp. upper) bound corresponds to progressive (resp. strictly progressive) strategy. Under Dynamic Programming Principle and identification of the generator, we can prove that the inf-sup type game is characterized as the unique viscosity solution of the Isaacs equation. We also discuss about the Isaacs equation with Hamiltonian of convex combination between lower and upper Hamiltonians.
\end{abstract}

\section{Introduction}

Let us consider dynamics of state $x(s) \in \mathbb{R}^{N}$ influenced by two factors $a, b$.

$$
\left\{\begin{aligned}
\frac{d x}{d s}(s) & =f(s, x(s), a(s), b(s)), t \leq s \leq T \\
x(t) & =x \in \mathbb{R}^{N}
\end{aligned}\right.
$$

where $a(\cdot), b(\cdot)$ are measurable functions on $[t, T]$ taking its value in metric space $A, B$ respectively. For given $a$ and $b$, we introduce game payoff function:

$$
J(t, x ; a, b) \equiv \int_{t}^{T} l(s, x(s), a(s), b(s)) d s+\Phi(x(T)),
$$

where $l:[0, T] \times \mathbb{R}^{N} \times A \times B \rightarrow \mathbb{R}, \Phi: \mathbb{R}^{N} \rightarrow \mathbb{R}$. In this problem, $a(\cdot)$ is considered as control of minimizing player and $b(\cdot)$ as control of maximizing player. Under certain assumption on available information for each player, we can define two types of game value.

$$
\begin{aligned}
V(t, x) & \equiv \inf _{\alpha} \sup _{b} J(t, x ; \alpha[b], b) \\
W(t, x) & \equiv \sup _{\beta} \inf _{a} J(t, x ; a, \beta[a]) .
\end{aligned}
$$

In (1.3), supremum is taken over any $b(\cdot)$ and infimum is taken on a set of mapping $\alpha$ from $b(\cdot)$ to $a(\cdot)$, which is considered as a class of strategies for minimizing player. (1.4) is interpreted in the same way. 
In conventional differential game, it is considered as a basic problem to find appropriate class of strategies which enable us to characterize $V, W$ and to identify $V$ with $W$ under min-max (Isaacs) condition. Evans and Souganidis [4] answered to these problems by using theory of viscosity solutions. In [4], by taking Elliott-Kalton strategy (see Definition 2.2 in the present paper) for strategy class, it is showed that Dynamic Programming Principles (DPPs) hold: For $0 \leq t<t+\delta \leq T, x \in \mathbb{R}^{N}$,

$$
\begin{gathered}
V(t, x)=\inf _{\alpha} \sup _{b}\left[\int_{t}^{t+\delta} l(s, x(s), \alpha[b](s), b(s)) d s+V(t+\delta, x(t+\delta))\right] \\
W(t, x)=\sup _{\beta} \inf _{a}\left[\int_{t}^{t+\delta} l(s, x(s), a(s), \beta[a](s)) d s+W(t+\delta, x(t+\delta))\right] .
\end{gathered}
$$

Then, by using DPPs, it is proved that $V$ and $W$ are characterized as the unique viscosity solution in the following Isaacs equations:

$$
\begin{gathered}
\left\{\begin{array}{c}
\frac{\partial V}{\partial t}(t, x)+\bar{H}(t, x, \nabla V(t, x))=0 \text { in }(0, T) \times \mathbb{R}^{N} \\
V(T, x)=\Phi(x), x \in \mathbb{R}^{N},
\end{array}\right. \\
\left\{\begin{array}{c}
\frac{\partial W}{\partial t}(t, x)+\underline{H}(t, x, \nabla W(t, x))=0 \text { in }(0, T) \times \mathbb{R}^{N} \\
W(T, x)=\Phi(x), x \in \mathbb{R}^{N},
\end{array}\right.
\end{gathered}
$$

where $\underline{H}, \bar{H}$ are defined as follows:

$$
\begin{aligned}
& \bar{H}(s, x, p)=\inf _{a \in A} \sup _{b \in B}[f(s, x, a, b) \cdot p+l(s, x, a, b)] \\
& \underline{H}(s, x, p)=\sup _{b \in B} \inf _{a \in A}[f(s, x, a, b) \cdot p+l(s, x, a, b)]
\end{aligned}
$$

Here, we point out that the order of inf and sup in DPPs is flipped in the Isaacs equation. Since comparison theorems for Isaacs equations are proved under some condition and $\underline{H} \leq \bar{H}$ holds, one can have

$$
V(t, x) \leq W(t, x)
$$

Note that under min-max condition $\underline{H}=\bar{H}$, we can see that $V(t, x)=W(t, x)$ because of uniqueness of viscosity solutions (see details in [4]).

In the above problem, both of minimizing and maximizing players are treated equally. On the other hand, we can give a special role to maximizing player. For instance, maximizing player is regarded as disturbance in $H^{\infty}$-control (cf. [2]). In this interpretation, inf-sup type value (1.3) is preferable to sup-inf type value (1.4). Recently, Fleming [5] considers inf-sup type value in terms of max-plus stochastic control which gives a generalization of $H^{\infty}$-theory. In [5], sup-inf type value $W$ in Elliott-Kalton sense is identified with inf-sup type value defined by smaller class of strategies than Elliott-Kalton strategies by using discretization method (see Theorem 4.1, [5]).

The aim of the present paper is to provide general framework how to relate infsup type games with the corresponding Isaacs equations. To utilize viscosity solution methods, we have to work with two steps: one is DPP and the other is identification of 
infinitesimal generator (cf. Chapter II, [7]). When we give a class of strategy, we can define a inf-sup type value by (1.3). Then, it would be natural to expect that DPP holds in the form of (1.5). If DPP (1.5) holds, we formally have the following equation:

$$
\lim _{\delta \rightarrow 0+} \frac{1}{\delta} \inf _{\alpha} \sup _{b}\left[\int_{t}^{t+\delta} l(s, x(s), \alpha[b](s), b(s)) d s+V(t+\delta, x(t+\delta))-V(t, x)\right]=0 .
$$

Indeed, this can be rewritten as follows:

$$
\lim _{\delta \rightarrow 0+} \frac{1}{\delta}\left(F_{t, t+\delta} V(t+\delta, \cdot)(x)-V(t, x)\right)=0
$$

where $F_{t, s}$ is defined for given function $\phi: \mathbb{R}^{N} \rightarrow \mathbb{R}$ as follows

$$
F_{t, s} \phi(x)=\inf _{\alpha} \sup _{b}\left[\int_{t}^{s} l(r, x(r), \alpha[b](r), b(r)) d r+\phi(x(s))\right], x \in \mathbb{R}^{N} .
$$

In general, it is known that $V$ is not smooth. So, we consider (1.9) for smooth function $\varphi(t, x)$ instead of $V$ :

$$
\lim _{\delta \rightarrow 0} \frac{1}{\delta}\left(F_{t, t+\delta} \varphi(t+\delta, \cdot)(x)-\varphi(t, x)\right)
$$

In Section 2, we shall study the infinitesimal generators (1.10). We give upper and lower bounds of (1.10) for quite general classes of strategies. In fact, lower bound is given by $\underline{H}$ and upper bound by $\bar{H}$. In general, it is not easy to find a class which has infinitesimal generator. However, we can show that the lower bound of generators corresponds to Elliott-Kalton strategy (which we call progressive in this paper) and the upper bound corresponds to strictly progressive strategy.

Relationships between DPP and infinitesimal generator are discussed in Section 3. Once the infinitesimal generator is obtained, we can consider the corresponding Isaacs equation. Following the general ideas of viscosity theory, we shall prove that if we have infinitesimal generator and DPP, the inf-sup value is a viscosity solution of the corresponding Isaacs equation. In this procedure, we also have to see if DPP holds. DPP for Elliott-Kalton case is proved in [4]. We shall show that DPP holds for strictly progressive strategy.

One of the interesting problems is to find a strategy class whose inf-sup value is characterized as viscosity solution of Isaacs equation with Hamiltonian different from $\underline{H}, \bar{H}$. In Section 3, we consider Isaacs equation with Hamiltonian given by convex combination of $\underline{H}, \bar{H}$. Instead of giving a strategy class, following the techniques of Souganidis in [9], [10], we consider approximated differential game. We prove that approximated value converges to viscosity solution of the Isaacs equation with the convex combination of the Hamiltonians.

Acknowledgement: The authors wish to thank Professor W.H. Fleming for valuable comments and suggestions. 


\section{Infinitesimal generators for game values}

As we mentioned in Introduction, it is an important step to study infinitesimal generators associated to game values. In this section, we shall give bounds for infinitesimal generators of inf-sup type value for general class of strategies. In particular, we shall identify infinitesimal generators for progressive and strictly progressive strategies.

In the rest of all the arguments, we always suppose the followings:

$A, B$ are compact subsets of Euclidean space.

$f, l$ and $\Phi$ are bounded and continuous.

There exists $L>0$ such that for each $s \in[0, T], x, y \in \mathbb{R}^{N}, a \in A, b \in B$

$$
\begin{gathered}
|f(s, x, a, b)-f(s, y, a, b)| \leq L|x-y| \\
|l(s, x, a, b)-l(s, y, a, b)| \leq L|x-y| \\
|\Phi(x)-\Phi(y)| \leq L|x-y| .
\end{gathered}
$$

Note that $f$ and $l$ are uniformly continuous on $[0, T] \times K \times A \times B$ for any compact set $K$.

$\mathcal{A}_{t, s}\left(\right.$ resp. $\left.\mathcal{B}_{t, s}\right)$ is the set of $A$-valued (resp. $B$-valued) measurable functions on $[t, s]$, which is considered as all of the controls for minimizing player (resp. maximizing player). We denote the set of mappings from $\mathcal{B}_{t, s}$ into $\mathcal{A}_{t, s}\left(\right.$ resp. $\mathcal{A}_{t, s}$ into $\left.\mathcal{B}_{t, s}\right)$ as $\Gamma_{t, s}^{0}$ (resp. $\left.\Delta_{t, s}^{0}\right)$. $\Gamma_{t, s}^{0}\left(\right.$ resp. $\left.\Delta_{t, s}^{0}\right)$ is considered as all the possible strategies of minimizing player (resp. maximizing player).

For given $\Gamma_{t, T} \subset \Gamma_{t, T}^{0}$, we define inf-sup type value:

$$
\begin{aligned}
V(t, x) & \equiv \inf _{\alpha \in \Gamma_{t, T}} \sup _{b \in \mathcal{B}_{t, T}} J(t, x ; \alpha[b], b) \\
& \equiv \inf _{\alpha \in \Gamma_{t, T}} \sup _{b \in \mathcal{B}_{t, T}}\left[\int_{t}^{T} l(s, x(s), \alpha[b](s), b(s)) d s+\Phi(x(T))\right],
\end{aligned}
$$

where $x(\cdot)$ is a solution of (1.1) with initial condition $x(t)=x$ and controls $a=\alpha[b], b$. We also introduce the operators on $C\left(\mathbb{R}^{N}\right)$ associated to $(2.1)$ :

$$
\begin{aligned}
F_{t, s}^{a, b} \phi(x) & =\int_{t}^{s} l(r, x(r), a(r), b(r)) d r+\phi(x(s)), \\
F_{t, s} \phi(x) & \equiv \inf _{\alpha \in \Gamma_{t, s}} \sup _{b \in \mathcal{B}_{t, s}} F_{t, s}^{a[b], b} \phi(x), \phi \in C\left(\mathbb{R}^{N}\right),
\end{aligned}
$$

where $x(\cdot)$ is a solution of $(1.1)$ with $x(t)=x$

Firstly, we obtain bounds for the generators in general case:

Proposition 2.1. Suppose $\Gamma_{t, s}$ includes open loop strategies, i.e., $\alpha: \mathcal{B}_{t, s} \rightarrow \mathcal{A}_{t, s}$ defined in the following belongs to $\Gamma_{t, s}$ :

$$
\alpha[b](r) \equiv a(r), t \leq r \leq s, b \in \mathcal{B}_{t, s} ; a \in \mathcal{A}_{t, s} .
$$


Then, for $\varphi \in C^{1}\left((0, T) \times \mathbb{R}^{N}\right)$, we have

$$
\begin{aligned}
& \varlimsup_{\delta \rightarrow 0+} \frac{1}{\delta}\left(F_{t_{\delta}, t_{\delta}+\delta} \varphi\left(t_{\delta}+\delta, \cdot\right)\left(x_{\delta}\right)-\varphi\left(t_{\delta}, x_{\delta}\right)\right) \leq \frac{\partial \varphi}{\partial t}(t, x)+\bar{H}\left(t, x, \nabla_{x} \varphi(t, x)\right), \\
& \frac{\partial \varphi}{\partial t}(t, x)+\underline{H}\left(t, x, \nabla_{x} \varphi(t, x)\right) \leq \varliminf_{\delta \rightarrow 0+}^{\lim } \frac{1}{\delta}\left(F_{t_{\delta}, t_{\delta}+\delta} \varphi\left(t_{\delta}+\delta, \cdot\right)\left(x_{\delta}\right)-\varphi\left(t_{\delta}, x_{\delta}\right)\right),
\end{aligned}
$$

where $\left(t_{\delta}, x_{\delta}\right)$ is any sequence such that $\left(t_{\delta}, x_{\delta}\right) \rightarrow(t, x) \in(0, T) \times \mathbb{R}^{N}$ as $\delta \rightarrow 0+$.

Proof. If we identify $a \in \mathcal{A}_{t, s}$ with the strategy defined in (2.3), we may consider $\mathcal{A}_{t, s} \subset \Gamma_{t, s}^{0}$. By the assumption that $\Gamma_{t, s}$ includes open loop strategies, $\mathcal{A}_{t, s} \subset \Gamma_{t, s}$. Then, we have

$$
\begin{aligned}
F_{t_{\delta}, t_{\delta}+\delta} \varphi\left(t_{\delta}+\delta, \cdot\right)\left(x_{t_{\delta}}\right) & =\inf _{\alpha \in \Gamma_{t_{\delta}, t_{\delta}+\delta}} \sup _{b \in \mathcal{B}_{t_{\delta}, t_{\delta}+\delta}} F_{t_{\delta}, t_{\delta}+\delta}^{\alpha[b], b} \varphi\left(t_{\delta}+\delta, \cdot\right)\left(x_{t_{\delta}}\right) \\
& \leq \inf _{a \in \mathcal{A}_{t_{\delta}, t_{\delta}+\delta}} \sup _{b \in \mathcal{B}_{t_{\delta}, t_{\delta}+\delta}} F_{t_{\delta}, t_{\delta}+\delta}^{a, b} \varphi\left(t_{\delta}+\delta, \cdot\right)\left(x_{t_{\delta}}\right) \\
& \leq \inf _{a \in A} \sup _{b \in \mathcal{B}_{t_{\delta}, t_{\delta}+\delta}} F_{t_{\delta}, t_{\delta}+\delta}^{a, b} \varphi\left(t_{\delta}+\delta, \cdot\right)\left(x_{t_{\delta}}\right) .
\end{aligned}
$$

For $a \in A$, we consider $a \in \mathcal{A}_{t, s}$ by $a(u)=a$. Let $a(u)=a \in \mathcal{A}_{t, s}$. By the definition of $F_{t_{\delta}, t_{\delta}+\delta}^{a, b}$,

$$
F_{t_{\delta}, t_{\delta}+\delta}^{a, b} \varphi\left(t_{\delta}+\delta, \cdot\right)\left(x_{t_{\delta}}\right)=\int_{t_{\delta}}^{t_{\delta}+\delta} l\left(s, x_{\delta}(s), a, b(s)\right) d s+\varphi\left(t_{\delta}+\delta, x_{\delta}\left(t_{\delta}+\delta\right)\right),
$$

where $x_{\delta}(\cdot)$ is a solution of (1.1) with initial condition $x_{\delta}\left(t_{\delta}\right)=x_{\delta}$. Note that for solution $x_{\delta}(\cdot)$ of $(1.1)$ with initial condition $x_{\delta}\left(t_{\delta}\right)=x_{\delta}$ driven by $a \in \mathcal{A}_{t_{\delta}, T}$ and $b \in \mathcal{B}_{t_{\delta}, T}$, there exists $K>0$ independent of $\delta, a, b$ such that

$$
\left|x_{\delta}(s)-x_{\delta}\right| \leq K s, t_{\delta} \leq \forall s \leq T
$$

By (A.1)-(A.3), (2.7), we can see that

$$
\begin{aligned}
& \int_{t_{\delta}}^{t_{\delta}+\delta} l\left(s, x_{\delta}(s), a, b(s)\right) d s+\varphi\left(t_{\delta}+\delta, x_{\delta}\left(t_{\delta}+\delta\right)\right)-\varphi\left(t_{\delta}, x_{\delta}\right) \\
& =\int_{t_{\delta}}^{t_{\delta}+\delta}\left[\frac{\partial \varphi}{\partial s}\left(s, x_{\delta}(s)\right)+f\left(s, x_{\delta}(s), a, b(s)\right) \cdot \nabla \varphi\left(s, x_{\delta}(s)\right)+l\left(s, x_{\delta}(s), a, b(s)\right)\right] d s \\
& =\int_{t_{\delta}}^{t_{\delta}+\delta}\left[\frac{\partial \varphi}{\partial s}\left(s, x_{\delta}\right)+f\left(s, x_{\delta}, a, b(s)\right) \cdot \nabla \varphi\left(s, x_{\delta}\right)+l\left(s, x_{\delta}, a, b(s)\right)\right] d s+o(\delta) \\
& =\frac{\partial \varphi}{\partial t}(t, x) \delta+\int_{t_{\delta}}^{t_{\delta}+\delta}[f(t, x, a, b(s)) \cdot \nabla \varphi(t, x)+l(t, x, a, b(s))] d s+o(\delta) \\
& \leq\left[\frac{\partial \varphi}{\partial t}(t, x)+\sup _{b \in B}\{f(t, x, a, b) \cdot \nabla \varphi(t, x)+l(t, x, a, b)\}\right] \delta+o(\delta)(\delta \rightarrow 0+)
\end{aligned}
$$


where $o(\delta)$ is uniform on $a$ and $b(\cdot)$. Thus, we have

$$
\begin{aligned}
& \quad \inf _{a \in A} \sup _{b \in \mathcal{B}_{t_{\delta}, t_{\delta}+\delta}} F_{t_{\delta}, t_{\delta}+\delta} \varphi\left(t_{\delta}+\delta, \cdot\right)\left(x_{\delta}\right)-\varphi\left(t_{\delta}, x_{\delta}\right) \\
& \quad \leq\left[\frac{\partial \varphi}{\partial t}(t, x)+\inf _{a \in A} \sup _{b \in B}\{f(t, x, a, b) \cdot \nabla \varphi(t, x)+l(t, x, a, b)\}\right] \delta+o(\delta)(\delta \rightarrow 0+) .
\end{aligned}
$$

Therefore, (2.4) implies from (2.6).

Fix arbitrary $\alpha \in \Gamma_{t_{\delta}, t_{\delta}+\delta}$ and $b \in \mathcal{B}_{t_{\delta}, t_{\delta}+\delta}$. Since $\mathcal{A}_{t_{\delta}, t_{\delta}+\delta} \subset \Gamma_{t_{\delta}, t_{\delta}+\delta}$ we have

$$
F_{t_{\delta}, t_{\delta}+\delta}^{\alpha[b], b} \varphi\left(t_{\delta}+\delta, \cdot\right)\left(x_{\delta}\right) \geq \inf _{a \in \mathcal{A}_{t_{\delta}, t_{\delta}+\delta}} F_{t_{\delta}, t_{\delta}+\delta}^{a, b} \varphi\left(t_{\delta}+\delta, \cdot\right)\left(x_{\delta}\right)
$$

By taking supremum over $b \in \mathcal{B}_{t_{\delta}, t_{\delta}+\delta}$ and then taking infimum over $\alpha \in \Gamma_{t_{\delta}, t_{\delta}+\delta}$,

$$
\begin{aligned}
\inf _{\alpha \in \Gamma_{t_{\delta}, t_{\delta}+\delta}} \sup _{b \in \mathcal{B}_{t_{\delta}, t_{\delta}+\delta}} F_{t_{\delta}, t_{\delta}+\delta}^{\alpha[b], b} \varphi\left(t_{\delta}+\delta, \cdot\right)\left(x_{\delta}\right) & \geq \sup _{b \in \mathcal{B}_{t_{\delta}, t_{\delta}+\delta}} \inf _{a \in \mathcal{A}_{t_{\delta}, t_{\delta}+\delta}} F_{t_{\delta}, t_{\delta}+\delta}^{a, b} \varphi\left(t_{\delta}+\delta, \cdot\right)\left(x_{\delta}\right) \\
& \geq \sup _{b \in B} \inf _{a \in \mathcal{A}_{t_{\delta}, t_{\delta}+\delta}} F_{t_{\delta}, t_{\delta}+\delta}^{a, b} \varphi\left(t_{\delta}+\delta, \cdot\right)\left(x_{\delta}\right) .
\end{aligned}
$$

In the same way as the proof of (2.4), we can prove that

$$
\begin{aligned}
& \sup _{b \in B} \inf _{a \in \mathcal{A}_{t_{\delta}, t_{\delta}+\delta}} F_{t_{\delta}, t_{\delta}+\delta}^{a, b} \varphi\left(t_{\delta}+\delta, \cdot\right)\left(x_{\delta}\right)-\varphi\left(t_{\delta}, x_{\delta}\right) \\
& \quad \geq\left[\frac{\partial \varphi}{\partial t}(t, x)+\sup _{b \in B} \inf _{a \in A}\{f(t, x, a, b) \cdot \nabla \varphi(t, x)+l(t, x, a, b)\}\right] \delta+o(\delta)(\delta \rightarrow 0+) .
\end{aligned}
$$

Then, by (2.8) and (2.9), we obtain (2.5).

In general, it is not obvious to find an explicit form of infinitesimal generators. However, we can identify the limit for progressive and strictly progressive strategies. We introduce these two notions of strategies.

Definition 2.2. (cf. [3], [8], [11]) $\alpha \in \Gamma_{t, s}^{0}$ is called progressive strategy for minimizing player if the following condition is satisfied:

$$
\text { For each } r \in[t, s] \text {, if } b \equiv \tilde{b} \text { a.e. on }[t, r] \text {, then } \alpha[b] \equiv \alpha[\tilde{b}] \text { a.e. on }[t, r] \text {. }
$$

We denote by $\Gamma_{t, s}^{P}$ the set of progressive strategies for minimizing player. Progressive strategy for maximizing player $\beta \in \Delta_{t, s}^{0}$ is defined in a similar way and the set of progressive strategies for maximizing player is denoted by $\Delta_{t, s}^{P}$.

Definition 2.3. (cf. [5]) $\alpha \in \Gamma_{t, s}^{P}$ is strictly progressive strategy if for any $\beta \in \Delta_{t, s}^{P}$, there exist $a \in \mathcal{A}_{t, s}$ and $b \in \mathcal{B}_{t, s}$ such that

$$
\alpha[b]=a, \beta[b]=b \text { a.e. on }[t, s] .
$$

We denote the set of strictly progressive strategies as $\Gamma_{t, s}^{S P}$.

Remark 2.4. $\Gamma_{t, s}^{P}$ and $\Gamma_{t, s}^{S P}$ include open loop strategies. 
We define the operators associated with these two classes of strategies:

$$
\begin{aligned}
& F_{t, s}^{P} \phi(x) \equiv \inf _{\alpha \in \Gamma_{t, s}^{P}} \sup _{b \in \mathcal{B}_{t, s}} F_{t, s}^{\alpha[b], b} \phi(x), \\
& F_{t, s}^{S P} \phi(x) \equiv \inf _{\alpha \in \Gamma_{t, s}^{S P}} \sup _{b \in \mathcal{B}_{t, s}} F_{t, s}^{\alpha[b], b} \phi(x)
\end{aligned}
$$

We also introduce the corresponding inf-sup type game values:

$$
\begin{aligned}
V^{P}(t, x) & \equiv \inf _{\alpha \in \Gamma_{t, T}^{P}} \sup _{b \in \mathcal{B}_{t, T}} J(t, x ; \alpha[b], b) \\
V^{S P}(t, x) & \equiv \inf _{\alpha \in \Gamma_{t, T}^{S P}} \sup _{b \in \mathcal{B}_{t, T}} J(t, x ; \alpha[b], b) .
\end{aligned}
$$

Note that under (A.1)-(A.3), $V^{P}$ and $V^{S P}$ are bounded and Lipschitz continuos on $[0, T] \times \mathbb{R}^{N}$

We first give the form of the generator for progressive case. The proof is implicitly done in [4]. However, we shall give another proof for convenience.

Proposition 2.5. For $\varphi \in C^{1}\left((0, T) \times \mathbb{R}^{N}\right)$,

$$
\frac{1}{\delta}\left(F_{t, t+\delta}^{P} \varphi(t+\delta, \cdot)(x)-\varphi(t, x)\right) \rightarrow \frac{\partial \varphi}{\partial t}(t, x)+\underline{H}(t, x, \nabla \varphi(t, x)), \delta \rightarrow 0+
$$

uniformly on each compact set in $(0, T) \times \mathbb{R}^{N}$.

Proof. Note that uniform convergence of (2.10) on each compact set is equivalent to the followings:

$$
\frac{1}{\delta}\left(F_{t_{\delta}+\delta, \delta}^{P} \varphi\left(t_{\delta}+\delta, \cdot\right)\left(x_{\delta}\right)-\varphi\left(t_{\delta}, x_{\delta}\right)\right) \rightarrow \frac{\partial \varphi}{\partial t}(t, x)+\underline{H}(t, x, \nabla \varphi(t, x))(\delta \rightarrow 0+)
$$

where $\left(t_{\delta}, x_{\delta}\right)$ is any sequence converging to $(t, x) \in(0, T) \times \mathbb{R}^{N}$. In Proposition 2.1, we already showed that

$$
\frac{\partial \varphi}{\partial t}(t, x)+\underline{H}(t, x, \nabla \varphi(t, x)) \leq \varliminf_{\delta \rightarrow 0+} \frac{1}{\delta}\left(F_{t_{\delta}+\delta, \delta}^{P} \varphi\left(t_{\delta}+\delta, \cdot\right)\left(x_{\delta}\right)-\varphi\left(t_{\delta}, x_{\delta}\right)\right) .
$$

So, we shall prove the other side of inequality

$$
\varlimsup_{\delta \rightarrow 0+} \frac{1}{\delta}\left(F_{t_{\delta}+\delta, \delta}^{P} \varphi\left(t_{\delta}+\delta, \cdot\right)\left(x_{\delta}\right)-\varphi\left(t_{\delta}, x_{\delta}\right)\right) \leq \frac{\partial \varphi}{\partial t}(t, x)+\underline{H}(t, x, \nabla \varphi(t, x)) .
$$

We take arbitrary $\theta>0$. Following the argument of Lemma 4.3 (b) in [4], we can construct a measurable mapping $\bar{a}: \mathcal{B} \rightarrow \mathcal{A}$ satisfying

$$
\begin{aligned}
& \frac{\partial \varphi}{\partial t}(t, x)+\underline{H}(t, x, \nabla \varphi(t, x)) \\
& \geq \frac{\partial \varphi}{\partial t}(t, x)+\inf _{a \in A}[f(t, x, a, b) \cdot \nabla \varphi(t, x)+l(t, x, a, b)] \\
& \geq \frac{\partial \varphi}{\partial t}(t, x)+f(t, x, \bar{a}(b), b) \cdot \nabla \varphi(t, x)+l(t, x, \bar{a}(b), b)-\theta, \forall b \in B .
\end{aligned}
$$


Define a strategy $\bar{\alpha} \in \Gamma_{t_{\delta}, t_{\delta}+\delta}^{0}$ as follows:

$$
\bar{\alpha}[b](s)=\bar{a}(b(s)), t_{\delta} \leq s \leq t_{\delta}+\delta ; b \in \mathcal{B}_{t_{\delta}, t_{\delta}+\delta} .
$$

In (2.12), if we take $\bar{a}=\bar{\alpha}[b](s), b=b(s)$ and integrate both of sides on $\left[t_{\delta}, t_{\delta}+\delta\right]$, we have

$$
\begin{aligned}
{\left[\frac{\partial \varphi}{\partial t}(t, x)+\underline{H}(t, x\right.} & , \nabla \varphi(t, x))] \delta \\
\geq \int_{t_{\delta}}^{t_{\delta}+\delta}\left(\frac{\partial \varphi}{\partial t}(t, x)\right. & +f(t, x, \bar{\alpha}[b](s), b(s)) \cdot \nabla \varphi(t, x) \\
& +l(t, x, \bar{\alpha}[b](s), b(s))) d s-\theta \delta, \forall b \in \mathcal{B}_{t_{\delta}, t_{\delta}+\delta}
\end{aligned}
$$

By (A.1)-(A.3) and (2.7), we can see that

$$
\begin{aligned}
& \int_{t_{\delta}}^{t_{\delta}+\delta}\left(\frac{\partial \varphi}{\partial t}(t, x)+f(t, x, \bar{\alpha}[b](s), b(s)) \cdot \nabla \varphi(t, x)+l(t, x, \bar{\alpha}[b](s), b(s))\right) d s \\
&=\int_{t_{\delta}}^{t_{\delta}+\delta}\left(\frac{\partial \varphi}{\partial t}\left(s, x_{\delta}(s)\right)+f\left(s, x_{\delta}(s), \bar{\alpha}[b](s), b(s)\right) \cdot \nabla \varphi\left(s, x_{\delta}(s)\right)\right. \\
&\left.+l\left(s, x_{\delta}(s), \bar{\alpha}[b](s), b(s)\right)\right) d s+o(\delta)
\end{aligned}
$$

where $x_{\delta}(\cdot)$ is a solution with $x_{\delta}\left(t_{\delta}\right)=x_{\delta}$ driven by $\bar{\alpha}[b], b$ and $o(\delta)$ is uniform on $\bar{\alpha}, b$ and $\theta$. Then, from (2.13) and (2.14), we have

$$
\begin{aligned}
& {\left[\begin{array}{l}
\left.\frac{\partial \varphi}{\partial t}(t, x)+\underline{H}(t, x, \nabla \varphi(t, x))\right] \delta \\
\geq \int_{t_{\delta}}^{t_{\delta}+\delta}\left(\frac{\partial \varphi}{\partial t}\left(s, x_{\delta}(s)\right)+f\left(s, x_{\delta}(s), \bar{\alpha}[b](s), b(s)\right) \cdot \nabla \varphi\left(s, x_{\delta}(s)\right)\right. \\
\left.\quad+l\left(s, x_{\delta}(s), \bar{\alpha}[b](s), b(s)\right)\right) d s+o(\delta)-\theta \delta
\end{array}\right.} \\
& =F_{t_{\delta}, t_{\delta}+\delta}^{\bar{\alpha}[b], b} \varphi\left(t_{\delta}+\delta, \cdot\right)\left(x_{\delta}\right)-\varphi\left(t_{\delta}, x_{t_{\delta}}\right)+o(\delta)-\theta \delta, \forall b \in \mathcal{B}_{t_{\delta}, t_{\delta}+\delta} .
\end{aligned}
$$

Since $b \in \mathcal{B}_{t_{\delta}, t_{\delta}+\delta}$ is arbitrary and $\bar{\alpha} \in \Gamma_{t_{\delta}, t_{\delta}+\delta}^{P}$,

$$
\left[\frac{\partial \varphi}{\partial t}(t, x)+\underline{H}(t, x, \nabla \varphi(t, x))\right] \delta \geq F_{t_{\delta}, t_{\delta}+\delta}^{P} \varphi\left(t_{\delta}+\delta, \cdot\right)\left(x_{\delta}\right)-\varphi\left(t_{\delta}, x_{t_{\delta}}\right)+o(\delta)-\theta \delta .
$$

By sending $\theta$ to 0 and dividing both of sides by $\delta$, we finally have

$$
\frac{\partial \varphi}{\partial t}(t, x)+\underline{H}(t, x, \nabla \varphi(t, x)) \geq \frac{1}{\delta}\left(F_{t_{\delta}, t_{\delta}+\delta}^{P} \varphi\left(t_{\delta}+\delta, \cdot\right)\left(x_{\delta}\right)-\varphi\left(t_{\delta}, x_{t_{\delta}}\right)\right)+o(1) .
$$

In the next result, we shall prove the infinitesimal generator associated to strictly progressive strategy corresponds to upper Hamiltonian $\bar{H}(t, x, p)$.

Proposition 2.6. For $\varphi \in C^{1}\left((0, T) \times \mathbb{R}^{N}\right)$,

$$
\frac{1}{\delta}\left(F_{t, t+\delta}^{S P} \varphi(t+\delta, \cdot)(x)-\varphi(t, x)\right) \rightarrow \frac{\partial \varphi}{\partial t}(t, x)+\bar{H}(t, x, \nabla \varphi(t, x)), \delta \rightarrow 0+
$$

uniformly on each compact set in $(0, T) \times \mathbb{R}^{N}$. 
Proof. As the proof of Proposition 2.5, it is enough to show that

$$
\frac{\partial \varphi}{\partial t}(t, x)+\bar{H}(t, x, \nabla \varphi(t, x)) \leq \varliminf_{\delta \rightarrow 0+} \frac{1}{\delta}\left(F_{t_{\delta}, t_{\delta}+\delta}^{S P} \varphi\left(t_{\delta}+\delta, \cdot\right)\left(x_{\delta}\right)-\varphi\left(t_{\delta}, x_{\delta}\right)\right)
$$

for any sequence $\left(t_{\delta}, x_{\delta}\right)$ converging to $(t, x) \in(0, T) \times \mathbb{R}^{N}$.

Take arbitrary $\theta>0$. In the same way as the argument in the proof of Proposition 2.5 (see the proof of Lemma 4.3 (b), [4]), there exists a measurable mapping $\bar{b}: \mathcal{A} \rightarrow \mathcal{B}$ such that

$$
\begin{aligned}
& \frac{\partial \varphi}{\partial t}(t, x)+\bar{H}(t, x, \nabla \varphi(t, x)) \\
& \leq \frac{\partial \varphi}{\partial t}(t, x)+\sup _{b \in B}[f(t, x, a, b) \cdot \nabla \varphi(t, x)+l(t, x, a, b)] \\
& \leq \frac{\partial \varphi}{\partial t}(t, x)+f(t, x, a, \bar{b}(a)) \cdot \nabla \varphi(t, x)+l(t, x, a, \bar{b}(a))+\theta, \forall a \in A .
\end{aligned}
$$

We define a new strategy $\bar{\beta} \in \Delta_{t_{\delta}, t_{\delta}+\delta}^{0}$ :

$$
\bar{\beta}[a](s)=\bar{b}(a(s)), t_{\delta} \leq s \leq t_{\delta}+\delta ; a \in \mathcal{A}_{t_{\delta}, t_{\delta}+\delta} .
$$

In fact, we can see that $\bar{\beta} \in \Delta_{t_{\delta}, t_{\delta}+\delta}^{P}$. By taking $a=a(s), b=\bar{\beta}[a](s)$ in (2.16) and integrating on $\left[t_{\delta}, t_{\delta}+\delta\right]$, we have

$$
\begin{aligned}
{\left[\frac{\partial \varphi}{\partial t}(t, x)+\bar{H}(t, x,\right.} & \nabla \varphi(t, x))] \delta \\
\leq \int_{t_{\delta}}^{t_{\delta}+\delta}\left(\frac{\partial \varphi}{\partial t}(t, x)+f(t, x, a(s), \bar{\beta}[a](s)) \cdot \nabla \varphi(t, x)\right. & +l(t, x, a(s), \bar{\beta}[a](s))) d s+\theta \delta, \forall a \in \mathcal{A}_{t_{\delta}, t_{\delta}+\delta} .
\end{aligned}
$$

From (A.1)-(A.3) and (2.7),

$$
\begin{aligned}
& \int_{t_{\delta}}^{t_{\delta}+\delta}\left(\frac{\partial \varphi}{\partial t}(\right.t, x)+f(t, x, a(s), \bar{\beta}[a](s)) \cdot \nabla \varphi(t, x)+l(t, x, a(s), \bar{\beta}[a](s))) d s \\
&=\int_{t_{\delta}}^{t_{\delta}+\delta}\left(\frac{\partial \varphi}{\partial t}\left(s, x_{\delta}(s)\right)+f\left(s, x_{\delta}(s), a(s), \bar{\beta}[a](s)\right) \cdot \nabla \varphi\left(s, x_{\delta}(s)\right)\right. \\
&\left.+l\left(s, x_{\delta}(s), a(s), \bar{\beta}[a](s)\right)\right) d s+o(\delta), \delta \rightarrow 0+
\end{aligned}
$$

where $o(\delta)$ is uniform on $a, \bar{\beta}, \theta$. Thus, we have

$$
\begin{aligned}
& \begin{array}{l}
{\left[\frac{\partial \varphi}{\partial t}(t, x)+\bar{H}(t, x, \nabla \varphi(t, x))\right] \delta} \\
\leq \int_{t_{\delta}}^{t_{\delta}+\delta}\left(\frac{\partial \varphi}{\partial t}\left(s, x_{\delta}(s)\right)+f\left(s, x_{\delta}(s), a(s), \bar{\beta}[a](s)\right) \cdot \nabla \varphi\left(s, x_{\delta}(s)\right)\right. \\
\left.\quad+l\left(s, x_{\delta}(s), a(s), \bar{\beta}[a](s)\right)\right) d s+o(\delta)+\theta \delta
\end{array} \\
& =F_{t_{\delta}, t_{\delta}+\delta}^{a, \bar{r}} \varphi\left(t_{\delta}+\delta, \cdot\right)\left(x_{\delta}\right)-\varphi\left(t_{\delta}, x_{\delta}\right)+o(\delta)+\theta \delta, \forall a \in \mathcal{A}_{t_{\delta}, t_{\delta}+\delta} .
\end{aligned}
$$


Take arbitrary $\alpha \in \Gamma_{t_{\delta}, t_{\delta}+\delta}^{S P}$. By the definition of strictly progressive strategy, there exist $\hat{a} \in \mathcal{A}_{t_{\delta}, t_{\delta}+\delta}$ and $\hat{b} \in \mathcal{B}_{t_{\delta}, t_{\delta}+\delta}$ such that

$$
\alpha[\hat{b}]=\hat{a}, \bar{\beta}[\hat{a}]=\hat{b} \quad \text { a.e. on }\left[t_{\delta}, t_{\delta}+\delta\right] .
$$

By (2.18) and (2.17), we have

$$
\begin{aligned}
{\left[\frac{\partial \varphi}{\partial t}(t, x)+\bar{H}(t, x, \nabla \varphi(t, x))\right] \delta } & \leq F_{t_{\delta}, t_{\delta}+\delta}^{\hat{a}, \bar{a}[\hat{a}]} \varphi\left(t_{\delta}+\delta, \cdot\right)\left(x_{\delta}\right)-\varphi\left(t_{\delta}, x_{\delta}\right)+o(\delta)+\theta \delta \\
& =F_{t_{\delta}, t_{\delta}+\delta}^{\alpha[\hat{b}] \hat{b}} \varphi\left(t_{\delta}+\delta, \cdot\right)\left(x_{\delta}\right)-\varphi\left(t_{\delta}, x_{\delta}\right)+o(\delta)+\theta \delta \\
& \leq \sup _{b \in \mathcal{B}_{t_{\delta}, t_{\delta}+\delta}} F_{t_{\delta}, t_{\delta}+\delta}^{\alpha[b], b} \varphi\left(t_{\delta}+\delta, \cdot\right)\left(x_{\delta}\right)-\varphi\left(t_{\delta}, x_{\delta}\right)+o(\delta)+\theta \delta
\end{aligned}
$$

Since $\alpha \in \Gamma_{t_{\delta}, t_{\delta}+\delta}$ is arbitrarily taken,

$$
\left[\frac{\partial \varphi}{\partial t}(t, x)+\bar{H}(t, x, \nabla \varphi(t, x))\right] \delta \leq F_{t_{\delta}, t_{\delta}+\delta}^{S P} \varphi\left(t_{\delta}+\delta, \cdot\right)\left(x_{\delta}\right)-\varphi\left(t_{\delta}, x_{\delta}\right)+o(\delta)+\theta \delta
$$

By taking $\theta \rightarrow 0$ and dividing by $\delta$, we obtain

$$
\frac{\partial \varphi}{\partial t}(t, x)+\bar{H}(t, x, \nabla \varphi(t, x)) \leq \frac{1}{\delta}\left(F_{t_{\delta}, t_{\delta}+\delta}^{S P} \varphi\left(t_{\delta}+\delta, \cdot\right)\left(x_{\delta}\right)-\varphi\left(t_{\delta}, x_{\delta}\right)\right)+o(1) .
$$

\section{Dynamic programming principle and Isaacs equa- tions}

In the present section, we shall study relationships between Dynamic Programming Principle (DPP) and its infinitesimal generators. More precisely, if DPP holds and the infinitesimal generator is identified, the inf-sup type game value is a viscosity solution of the corresponding Isaacs equation. Furthermore, if the Hamiltonian of the Isaacs equation satisfies certain structural conditions, the value is characterized as the unique viscosity solution.

In progressive and strictly progressive case, we can show DPPs hold. By combining results in Section 2, we shall prove that value defined by progressive (resp. strictly progressive) strategy is characterized as a unique viscosity solution for lower Isaacs equation (resp. upper Isaacs equation).

Firstly, we give a general result on relationship between DPP and the Isaacs equation. For given class of strategies $\Gamma_{t, s} \subset \Gamma_{t, s}^{0}, 0 \leq t<s \leq T$, we define inf-sup type game value $V(t, x)$ as (2.1). For given class $\Gamma_{t, s}$, DPP is described as follows: For $0 \leq t<t+\delta \leq T$, $x \in \mathbb{R}^{N}$,

$$
V(t, x)=\inf _{\alpha \in \Gamma_{t, t+\delta}} \sup _{b \in \mathcal{B}_{t, t+\delta}}\left[\int_{t}^{t+\delta} l(s, x(s), \alpha[b](s), b(s)) d s+V(t+\delta, x(t+\delta))\right] .
$$

This is reformulated in terms of (2.2):

$$
F_{t, t+\delta} V(t+\delta, \cdot)(x)=V(t, x)
$$


Proposition 3.1. Suppose that (3.1) holds and the infinitesimal generator is identified, i.e., there exists $H(t, x, p)$ such that for $\varphi \in C^{1}\left((0, T) \times \mathbb{R}^{N}\right)$,

$$
\frac{1}{\delta}\left(F_{t, t+\delta} \varphi(t+\delta, \cdot)(x)-\varphi(t, x)\right) \rightarrow \frac{\partial \varphi}{\partial t} \varphi(t, x)+H(t, x, \nabla \varphi(t, x)), \delta \rightarrow 0+.
$$

Then, $V(t, x)$ is a viscosity solution of the equation:

$$
\left\{\begin{array}{c}
\frac{\partial V}{\partial t}(t, x)+H(t, x, \nabla V(t, x))=0,(t, x) \in(0, T) \times \mathbb{R}^{N} \\
V(T, x)=\Phi(x), x \in \mathbb{R}^{N}
\end{array}\right.
$$

Proof. Suppose that $\varphi$ is smooth function on $(0, T) \times \mathbb{R}^{N}$ and $(t, x) \in(0, T) \times \mathbb{R}^{N}$ is a local maximum point of $V-\varphi$. We note that for sufficiently small $\delta>0$,

$$
F_{t, t+\delta} \varphi(t, x)-\varphi(t, x) \geq 0
$$

In fact, since $(t, x)$ is a local maximum point of $V-\varphi$, there exists $r>0$ such that

$$
V(s, y)-\varphi(s, y) \leq V(t, x)-\varphi(t, x), \forall(t, x) \in[t-r, t+r] \times \bar{B}_{r}(x)
$$

i.e.

$$
V(s, y)-V(t, x) \leq \varphi(s, y)-\varphi(t, x), \forall(t, x) \in[t-r, t+r] \times \bar{B}_{r}(x) .
$$

By the definition of $F_{t, t+\delta}$ and (3.1),

$$
\inf _{\alpha \in \Gamma_{t, t+\delta}} \sup _{b \in \mathcal{B}_{t, t+\delta}}\left[\int_{t}^{t+\delta} l(s, x(s), \alpha[b](s), b(s)) d s+V(t+\delta, x(t+\delta))-V(t, x)\right]=0 .
$$

By continuity of solution on initial point (2.7), we can take sufficiently small $\delta_{0}>0$ uniformly on $\alpha$ and $b$ such that

$$
x(s) \in B_{r}(x), t \leq s \leq t+\delta_{0} .
$$

Then, from (3.5) and (3.6), we obtain (3.4).

By (3.2) and (3.4), we have

$$
\frac{\partial \varphi}{\partial t}(t, x)+H(t, x, \nabla \varphi(t, x)) \geq 0 .
$$

Thus, $V$ is a viscosity subsolution of (3.3).

The proof of viscosity supersolution is the same as the above argument.

We shall next apply Proposition 3.1 to progressive and strictly progressive cases. In Section 2, we proved that infinitesimal generators for progressive strategies and strictly progressive strategies have explicit forms. To associate the values defined by these classes with the corresponding Isaacs equation, we need to prove DPP.

In progressive case, DPP and characterization of $V^{P}(t, x)$ as viscosity solution is proved in [4] (See Theorems 3.1, 4.1, [4]). By using DPP in [4], we can apply Propositions 2.5 and 3.1 to obtain characterization result for $V^{P}$. 
Proposition 3.2 (Theorem 3.1, [4]). $V^{P}(t, x)$ satisfies DPP:

$$
\begin{aligned}
V^{P}(t, x) & =F_{t, t+\delta}^{P} V^{P}(t+\delta, \cdot)(x) \\
& =\inf _{\alpha \in \Gamma_{t, t+\delta}^{P}} \sup _{b \in \mathcal{B}_{t, t+\delta}}\left[\int_{t}^{t+\delta} l(s, x(s), \alpha[b](s), b(s)) d s+V^{P}(t+\delta, x(s))\right] .
\end{aligned}
$$

We have another proof for characterization of $V^{P}$.

Theorem 3.3 (cf. Theorem 4.1, $[4]) . V^{P}(t, x)$ is unique viscosity solution in $B U C([0, T] \times$ $\left.\mathbb{R}^{N}\right)$ for the lower Isaacs equation:

$$
\left\{\begin{array}{c}
\frac{\partial V^{P}}{\partial t}(t, x)+\underline{H}\left(t, x, \nabla V^{P}(t, x)\right)=0,(t, x) \in(0, T) \times \mathbb{R}^{N}, \\
V^{P}(T, x)=\Phi(x), x \in \mathbb{R}^{N},
\end{array}\right.
$$

where $B U C\left([0, T] \times \mathbb{R}^{N}\right)$ is the set of bounded, Lipschitz continuous functions on $[0, T] \times$ $\mathbb{R}^{N}$.

Proof. By Propositions 2.5 and 3.2, we can apply Proposition 3.1 to show that $V^{P}(t, x)$ is a viscosity solution of (3.7). Under (A.1)-(A.3), Hamiltonian $\underline{H}$ satisfies structural condition to ensure uniqueness results in $B U C\left([0, T] \times \mathbb{R}^{N}\right)$ (cf. Theorem 9.1, Chapter II, [7], Exercise 3.9, Chapter II, [1]).

We consider value for strictly progressive strategies $V^{S P}(t, x)$. In [5], relationship between $V^{S P}$ and upper Isaacs equation is studied and $V^{S P}$ is identified with upper game value which is a viscosity solution of upper Isaacs equation. On the other hand, we directly prove DPP and apply Propositions 2.6 and 3.1.

Proposition 3.4. $V^{S P}(t, x)$ satisfies DPP: For $0 \leq t<t+\delta \leq T, x \in \mathbb{R}^{N}$,

$$
\begin{aligned}
V^{S P}(t, x) & =F_{t, t+\delta}^{S P} V^{S P}(t+\delta, \cdot)(x) \\
& =\inf _{\alpha \in \Gamma_{t, t+\delta}^{S P}} \sup _{b \in \mathcal{B}_{t, t+\delta}}\left[\int_{t}^{t+\delta} l(s, x(s), \alpha[b](s), b(s)) d s+V^{S P}(t+\delta, x(t+\delta))\right] .
\end{aligned}
$$

Proof. Set $W(t, x)=F_{t, t+\delta}^{S P} V^{S P}(t+\delta, \cdot)(x)$. We shall first show that $W(t, x) \geq V^{S P}(t, x)$. For any $\epsilon>0$, there exists $\alpha^{\epsilon} \in \Gamma_{t, t+\delta}^{S P}$ such that

$$
\begin{aligned}
W(t, x) & \geq \sup _{b_{1} \in \mathcal{B}_{t, t+\delta}} F_{t, t+\delta}^{\alpha^{\epsilon}\left[b_{1}\right], b_{1}} V^{S P}(t+\delta, \cdot)(x)-\epsilon \\
& \geq \int_{t}^{t+\delta} l\left(s, x_{1}^{\epsilon}(s), \alpha^{\epsilon}\left[b_{1}\right](s), b_{1}(s)\right) d s+V^{S P}\left(t+\delta, x_{1}^{\epsilon}(t+\delta)\right)-\epsilon, \forall b_{1} \in \mathcal{B}_{t, t+\delta},
\end{aligned}
$$

where $x_{1}^{\epsilon}(\cdot)$ is a solution of $(1.1)$ controlled by $\alpha^{\epsilon}\left[b_{1}\right]$ and $b_{1}$. We consider $V^{S P}(t+\delta, z)$, $z \in \mathbb{R}^{N}$. By definition of $V^{S P}(t+\delta, z)$, there exists $\alpha_{z}^{\epsilon} \in \Gamma_{t+\delta, T}^{S P}$ such that

$$
\begin{aligned}
V(t+\delta, z) & \geq \sup _{b_{2} \in \mathcal{B}_{t+\delta, T}} F_{t+\delta, T}^{\alpha_{z}^{\epsilon}\left[b_{2}\right], b_{s}} \Phi(z)-\epsilon \\
& \geq \int_{t+\delta}^{T} l\left(s, x_{2}^{\epsilon}(s), \alpha_{z}^{\epsilon}\left[b_{2}\right](s), b_{2}(s)\right) d s+\Phi\left(x_{2}^{\epsilon}(T)\right)-\epsilon, \forall b_{2} \in \mathcal{B}_{t+\delta, T},
\end{aligned}
$$


where $x_{2}^{\epsilon}(\cdot)$ is a solution of $(1.1)$ with initial condition $x_{2}^{\epsilon}(t+\delta)=z$, controls $\alpha_{z}^{\epsilon}\left[b_{2}\right], b_{2}$.

Define a new strategy $\bar{\alpha}^{\epsilon}: \mathcal{B}_{t, T} \rightarrow \mathcal{A}_{t, T}$ : For $b \in \mathcal{B}_{t, T}$,

$$
\bar{\alpha}^{\epsilon}[b](s)=\left\{\begin{array}{l}
\alpha^{\epsilon}\left[\left.b\right|_{[t, t+\delta]}\right](s), t \leq s<t+\delta \\
\alpha_{x_{1}^{\epsilon}(t+\delta)}^{\epsilon}\left[\left.b\right|_{[t+\delta, T]}\right](s), t+\delta \leq s \leq T
\end{array}\right.
$$

where $x_{1}^{\epsilon}(\cdot)$ is a solution of $(1.1)$ with controls $\alpha^{\epsilon}\left[\left.b\right|_{[t, t+\delta]}\right],\left.b\right|_{[t, t+\delta]}$. We used the notation $\left.b\right|_{[s, r]}$ for restriction of $b$ on $[s, r]$.

We shall show that $\bar{\alpha}^{\epsilon} \in \Gamma_{t, T}^{S P}$. Take arbitrary $\beta \in \Delta_{t, T}^{P}$. For given $\hat{a}_{2} \in \mathcal{A}_{t+\delta, T}$, define $\beta_{1}: \mathcal{A}_{t, t+\delta} \rightarrow \mathcal{B}_{t, t+\delta}$ as follows:

$$
\beta_{1}\left[a_{1}\right](s)=\beta\left[a_{1} \oplus \hat{a}_{2}\right](s), t \leq s \leq t+\delta, a_{1} \in \mathcal{A}_{t, t+\delta} .
$$

Here $a_{1} \oplus \hat{a}_{2}$ is defined by

$$
a_{1} \oplus \hat{a}_{2}=\left\{\begin{array}{l}
a_{1}(s), t \leq s<t+\delta \\
\hat{a}_{2}(s), t+\delta \leq s \leq T .
\end{array}\right.
$$

Note that $\beta_{1}$ does not depend on $\hat{a}_{2} \in \mathcal{A}_{t+\delta, T}$ because $\beta_{1}\left[a_{1}\right]=\left.\beta\left[a_{1} \oplus \hat{a}_{2}\right]\right|_{[t, t+\delta]}$ is determined by $a_{1}$ on $[t, t+\delta]$ (See Definition 2.2). Since $\alpha^{\epsilon} \in \Gamma_{t, t+\delta}^{S P}$, there exist $\bar{a}_{1} \in \mathcal{A}_{t, t+\delta}$, $\bar{b}_{1} \in \mathcal{B}_{t, t+\delta}$ such that

$$
\alpha^{\epsilon}\left[\bar{b}_{1}\right]=\bar{a}_{1}, \beta_{1}\left[\bar{a}_{1}\right]=\bar{b}_{1} \text { on }[t, t+\delta] .
$$

Let $\bar{x}_{1}^{\epsilon}(\cdot)$ be solution of (1.1) by $\alpha^{\epsilon}\left[\bar{b}_{1}\right], \bar{b}_{1}$. Define $\beta_{2}: \mathcal{A}_{t+\delta, T} \rightarrow \mathcal{B}_{t+\delta, T}$ in the following:

$$
\beta_{2}\left[a_{2}\right](s)=\beta\left[\bar{a}_{1} \oplus a_{2}\right](s), t+\delta \leq s \leq T, a_{2} \in \mathcal{A}_{t+\delta, T} .
$$

Note that $\beta_{2} \in \Delta_{t+\delta, T}^{P}$. It implies from $\alpha_{\bar{x}_{1}^{\epsilon}(t+\delta)}^{\epsilon} \in \Gamma_{t+\delta, T}^{S P}$ that there exist $\bar{a}_{2} \in \mathcal{A}_{t+\delta, T}$, $\bar{b}_{2} \in \mathcal{B}_{t+\delta, T}$ such that

$$
\alpha_{\bar{x}_{1}^{\epsilon}(t+\delta)}^{\epsilon}\left[\bar{b}_{2}\right]=\bar{a}_{2}, \beta_{2}\left[\bar{a}_{2}\right]=\bar{b}_{2} \text { on }[t+\delta, T] .
$$

If we set $\bar{a}=\bar{a}_{1} \oplus \bar{a}_{2}, \bar{b}=\bar{b}_{1} \oplus \bar{b}_{2}$, then we have from (3.11), (3.13), (3.14),

$$
\bar{\alpha}^{\epsilon}[\bar{b}]=\bar{a}, \beta[\bar{a}]=\bar{b} \text { on }[t, T] .
$$

Therefore we proved that $\bar{\alpha}^{\epsilon} \in \Gamma_{t, T}^{S P}$.

By definition of $\alpha^{\epsilon},(3.9),(3.10)$,

$$
\begin{aligned}
W(t, x) \geq & \int_{t}^{t+\delta} l\left(s, \bar{x}^{\epsilon}(s), \bar{\alpha}^{\epsilon}[b](s), b(s)\right) d s \\
& \quad+\int_{t+\delta}^{T} l\left(s, \bar{x}^{\epsilon}(s), \bar{\alpha}^{\epsilon}[b](s), b(s)\right) d s+\Phi\left(x^{\epsilon}(T)\right)-2 \epsilon \\
& \geq J\left(t, x ; \bar{\alpha}^{\epsilon}[b], b\right)-2 \epsilon, \quad \forall b \in \mathcal{B}_{t, T}
\end{aligned}
$$


where $\bar{x}^{\epsilon}(\cdot)$ is a solution of (1.1) with $\bar{\alpha}^{\epsilon}[b], b$. Thus, we have

$$
W(t, x) \geq \inf _{\alpha \in \Gamma_{t, T}^{S P}} \sup _{b \in \mathcal{B}_{t, T}} J(t, x ; \alpha[b], b)-2 \epsilon=V^{S P}(t, x)-2 \epsilon .
$$

Since $\epsilon>0$ is arbitrary, we finally obtain

$$
W(t, x) \geq V^{S P}(t, x) .
$$

We next show that $W(t, x) \leq V^{S P}(t, x)$. Fix arbitrary $\alpha \in \Gamma_{t, T}^{S P}$. For given $\tilde{b}_{2} \in \mathcal{B}_{t+\delta, T}$, we introduce a strategy $\alpha_{1}: \mathcal{B}_{t, t+\delta} \rightarrow \mathcal{A}_{t, t+\delta}$ :

$$
\alpha_{1}\left[b_{1}\right](s) \equiv \alpha\left[b_{1} \oplus \tilde{b}_{2}\right](s), t \leq s \leq t+\delta, b_{1} \in \mathcal{B}_{t, t+\delta} .
$$

Note that $\alpha_{1}$ does not depend on the choice of $\tilde{b}_{2}$ because $\alpha \in \Gamma_{t, T}^{P}$.

We shall see that $\alpha_{1} \in \Gamma_{t, t+\delta}^{S P}$. For given $\beta_{1} \in \Delta_{t, t+\delta}^{P}$, we can find $\beta \in \Delta_{t, T}^{P}$ such that

$$
\beta[a]=\beta_{1}\left[\left.a\right|_{[t, t+\delta]}\right] \text { on }[t, t+\delta], a \in \mathcal{A}_{t, T} .
$$

For instance, we can construct $\beta$ as follows:

$$
\beta[a](s)=\left\{\begin{array}{l}
\beta_{1}\left[\left.a\right|_{[t, t+\delta]}\right](s), t \leq s<t+\delta \\
b^{0}, t \leq s \leq T
\end{array}\right.
$$

where $b^{0} \in B$ is a fixed constant. Since $\alpha \in \Gamma_{t, T}^{S P}$, there exist $\hat{a} \in \mathcal{A}_{t, T}, \hat{b} \in \mathcal{B}_{t, T}$ such that

$$
\alpha[\hat{b}]=\hat{a}, \beta[\hat{a}]=\hat{b} \text { on }[t, T]
$$

In (3.15), if we take $\tilde{b}_{2}=\left.\hat{b}\right|_{[t+\delta, T]}$, we have from (3.16)

$$
\alpha_{1}\left[\hat{b}_{1}\right]=\hat{a}_{1}, \beta_{1}\left[\hat{a}_{1}\right]=\hat{b}_{1} \text { on }[t, t+\delta]
$$

where $\hat{a}_{1}=\left.\hat{a}\right|_{[t, t+\delta]}, \hat{b}_{1}=\left.\hat{b}\right|_{[t, t+\delta]}$. Hence, $\alpha_{1} \in \Gamma_{t, t+\delta}^{S P}$.

By definition of $W(t, x)$ and $\alpha_{1} \in \Gamma_{t, t+\delta}^{S P}$,

$$
W(t, x) \leq \sup _{b_{1} \in \mathcal{B}_{t, t+\delta}}\left[\int_{t}^{t+\delta} l\left(s, x_{1}(s), \alpha_{1}\left[b_{1}\right](s), b_{1}(s)\right) d s+V^{S P}\left(t+\delta, x_{1}(t+\delta)\right)\right] .
$$

For any $\epsilon>0$, there exists $b_{1}^{\epsilon} \in \mathcal{B}_{t, t+\delta}$ such that

$$
W(t, x) \leq \int_{t}^{t+\delta} l\left(s, x_{1}^{\epsilon}(s), \alpha_{1}\left[b_{1}^{\epsilon}\right](s), b_{1}^{\epsilon}(s)\right) d s+V^{S P}\left(t+\delta, x_{1}^{\epsilon}(t+\delta)\right)+\epsilon
$$

where $x_{1}^{\epsilon}(\cdot)$ is solution of $(1.1)$ controlled by $\alpha_{1}\left[b_{1}^{\epsilon}\right], b_{1}^{\epsilon}$.

Define $\alpha_{2}: \mathcal{B}_{t+\delta, T} \rightarrow \mathcal{A}_{t+\delta, T}$ as follows:

$$
\alpha_{2}\left[b_{2}\right](s) \equiv \alpha\left[b_{1}^{\epsilon} \oplus b_{2}\right](s), t+\delta \leq s \leq T, b_{2} \in \mathcal{B}_{t+\delta, T}
$$


In order to prove that $\alpha_{2} \in \Gamma_{t+\delta, T}^{S P}$, we take any $\hat{\beta}_{2} \in \Delta_{t+\delta, T}^{P}$. Define $\hat{\beta}: \mathcal{A}_{t, T} \rightarrow \mathcal{B}_{t, T}$ :

$$
\hat{\beta}[a](s) \equiv\left\{\begin{array}{l}
b_{1}^{\epsilon}(s), t \leq s<t+\delta \\
\hat{\beta}_{2}\left[\left.a\right|_{[t+\delta, T]}\right], t+\delta \leq s \leq T .
\end{array}\right.
$$

It is easy to see that $\hat{\beta} \in \Delta_{t, T}^{P}$. Since $\alpha \in \Gamma_{t, T}^{S P}$, there exist $\bar{a} \in \mathcal{A}_{t, T}$ and $\bar{b} \in \mathcal{B}_{t, T}$ such that

$$
\alpha[\bar{b}]=\bar{a}, \hat{\beta}[\bar{a}]=\bar{b} \text { on }[t, T] .
$$

From definition of $\hat{\beta}$, we see that $b_{1}^{\epsilon}=\left.\hat{\beta}[\bar{a}]\right|_{[t, t+\delta]}=\bar{b}$ on $[t, t+\delta]$. Thus, we have

$$
\bar{b}=\left.b_{1}^{\epsilon} \oplus \bar{b}\right|_{[t+\delta, T]} .
$$

Thus, if we set $\bar{a}_{2}=\left.\bar{a}\right|_{[t+\delta, T]}, \bar{b}_{2}=\left.\bar{b}\right|_{[t+\delta, T]}$, we have

$$
\alpha_{2}\left[\bar{b}_{2}\right]=\bar{a}_{2}, \hat{\beta}\left[\bar{a}_{2}\right]=\bar{b}_{2} \text { on }[t+\delta, T] .
$$

Hence $\alpha_{2} \in \Gamma_{t+\delta, T}^{S P}$.

From definition of $V^{S P}\left(t+\delta, x_{1}^{\epsilon}(t+\delta)\right)$,

$$
V^{S P}\left(t+\delta, x_{1}^{\epsilon}(t+\delta)\right) \leq \sup _{b_{2} \in \mathcal{B}_{t+\delta, T}}\left[\int_{t+\delta}^{T} l\left(s, x_{2}(s), \alpha_{2}\left[b_{2}\right](s), b_{2}(s)\right) d s+\Phi\left(x_{2}(T)\right)\right]+\epsilon .
$$

For $\epsilon>0$, there exists $b_{2}^{\epsilon} \in \mathcal{B}_{t+\delta, T}$ such that

$$
\left.V^{S P}\left(t+\delta, x_{1}^{\epsilon}(t+\delta)\right) \leq \int_{t+\delta}^{T} l\left(s, x_{2}^{\epsilon}(s), \alpha_{2}\left[b_{2}^{\epsilon}\right](s), b_{2}^{\epsilon}(s)\right) d s+\Phi\left(x_{2}^{\epsilon}(T)\right)\right]
$$

where $x_{2}^{\epsilon}(\cdot)$ is solution with initial condition $x_{2}^{\epsilon}(t+\delta)=x_{1}^{\epsilon}(t+\delta)$ controlled by $\alpha_{2}\left[b_{2}^{\epsilon}\right], b_{2}^{\epsilon}$. We set $b^{\epsilon}=b_{1}^{\epsilon} \oplus b_{2}^{\epsilon}$. Then, by (3.17), (3.18),

$$
\begin{aligned}
W(t, x) \leq & \int_{t}^{t+\delta} l\left(s, x_{1}^{\epsilon}(s), \alpha\left[b_{1}^{\epsilon} \oplus b_{2}^{\epsilon}\right](s), b_{1}^{\epsilon}(s)\right) d s \\
& \quad+\int_{t+\delta}^{T} l\left(s, x_{2}^{\epsilon}(s), \alpha\left[b_{1} \oplus b_{2}^{\epsilon}\right](s), b_{2}^{\epsilon}(s)\right) d s+\Phi\left(x_{2}^{\epsilon}(T)\right)+2 \epsilon \\
= & \int_{t}^{T} l\left(s, x^{\epsilon}(s), \alpha\left[b^{\epsilon}\right](s), b^{\epsilon}(s)\right) d s+\Phi\left(x^{\epsilon}(T)\right)+2 \epsilon \\
= & J\left(t, x ; \alpha\left[b^{\epsilon}\right], b^{\epsilon}\right)+2 \epsilon
\end{aligned}
$$

where $x^{\epsilon}(\cdot)$ is solution of (1.1) with $\alpha\left[b^{\epsilon}\right], b^{\epsilon}$. Since $\alpha \in \Gamma_{t, T}^{S P}$ is arbitrarily taken, we have

$$
W(t, x) \leq \inf _{\alpha \in \Gamma_{t, T}^{S P}} \sup _{b \in \mathcal{B}_{t, T}} J(t, x ; \alpha[b], b)+2 \epsilon=V^{S P}(t, x)+2 \epsilon .
$$

Taking $\epsilon \rightarrow 0$,

$$
W(t, x) \leq V^{S P}(t, x)
$$

Then, we have characterization for $V^{S P}$. 
Theorem 3.5. $V^{S P}(t, x)$ is the unique viscosity solution in $B U C\left([0, T] \times \mathbb{R}^{N}\right)$ for the upper Isaacs equation:

$$
\left\{\begin{array}{c}
\frac{\partial V^{S P}}{\partial t}(t, x)+\bar{H}\left(t, x, \nabla V^{S P}(t, x)\right)=0,(t, x) \in(0, T) \times \mathbb{R}^{N} \\
V^{S P}(T, x)=\Phi(x), x \in \mathbb{R}^{N} .
\end{array}\right.
$$

Proof. By Propositions 2.6, 3.1, 3.4, $V^{S P}$ is a viscosity solution of (3.19). By uniqueness or comparison theorem in the class $B U C\left([0, T] \times \mathbb{R}^{N}\right), V^{S P}$ is the unique viscosity solution (See [1], [7] ).

\section{Approximations of games for general Hamiltonians}

For class of strategies $\Gamma_{t, s}$ such that $\Gamma_{t, s}^{S P} \subset \Gamma_{t, s} \subset \Gamma_{t, s}^{P}$, we can introduce an inf-sup type value:

$$
V(t, x) \equiv \inf _{\alpha \in \Gamma_{t, T}} \sup _{b \in \mathcal{B}_{t, T}} J(t, x ; \alpha[b], b) .
$$

Note that $V^{P}(t, x) \leq V(t, x) \leq V^{S P}(t, x)$. In the previous sections, we gave a general framework to relate DPP and the corresponding Isaacs equations. Particularly, $V^{P}$ (resp. $V^{S P}$ ) is characterized as the unique viscosity solution of lower Isaacs equation (resp. upper Isaacs equation). As Proposition 2.1 indicates, it is an interesting problem to study some class $\Gamma_{t, s}$ for which the infinitesimal generator is different from $\underline{H}$ and $\bar{H}$ and $V(t, x)$ satisfies DPP. However, it seems not easy to find such class immediately.

In this section, we consider an approximation problem for Hamiltonian $H^{(\gamma)}$ of convex combination between $\underline{H}$ and $\bar{H}$ :

$$
H^{(\gamma)}(t, x, p)=(1-\gamma) \underline{H}(t, x, p)+\gamma \bar{H}(t, x, p), 0 \leq \gamma \leq 1
$$

We shall introduce an approximation of game problem by following techniques of Souganidis in [9], [10]. We use the product of $F_{t, s}^{P}, F_{t, s}^{S P}$ with some weight related to $\gamma$ to define discrete game problem. Then, by taking the limit with respect to the size of sub-interval, we shall show that the discrete game value converges to the unique viscosity solution of (3.3) with Hamiltonian $H=H^{(\gamma)}$.

For $0 \leq \gamma \leq 1$, we define operator $F_{t, s}^{(\gamma)}$ as follows:

$$
F_{t, s}^{(\gamma)} \phi(x) \equiv F_{t, t+\gamma(s-t)}^{S P} F_{t+\gamma(s-t), s}^{P} \phi(x), x \in \mathbb{R}^{N}, 0 \leq t \leq s ; \phi \in C\left(\mathbb{R}^{N}\right) .
$$

At first, we obtain the result on infinitesimal generator for (4.1).

Proposition 4.1. For $\varphi \in C^{1}\left((0, T) \times \mathbb{R}^{N}\right)$,

$$
\frac{1}{\delta}\left(F_{t, t+\delta}^{(\gamma)} \varphi(t+\delta, \cdot)(x)-\varphi(t, x)\right) \rightarrow \frac{\partial \varphi}{\partial t}(t, x)+H^{(\gamma)}(t, x, \nabla \varphi(t, x)), \delta \rightarrow 0+
$$

uniformly on each compact set in $(0, T) \times \mathbb{R}^{n}$. 
Proof. It is enough to show that for each sequence $\left(t_{\delta}, x_{\delta}\right)$ converging to $(t, x) \in(0, T) \times$ $\mathbb{R}^{N}$,

$$
\frac{1}{\delta}\left(F_{t_{\delta}, t_{\delta}+\delta}^{(\gamma)} \varphi\left(t_{\delta}+\delta, \cdot\right)\left(x_{\delta}\right)-\varphi\left(t_{\delta}, x_{\delta}\right)\right) \rightarrow \frac{\partial \varphi}{\partial t}(t, x)+H^{(\gamma)}(t, x, \nabla \varphi(t, x)), \delta \rightarrow 0+.
$$

From (4.1), we have

$$
\begin{aligned}
& F_{t_{\delta}, t_{\delta}+\delta}^{(\gamma)} \varphi\left(t_{\delta}+\delta, \cdot\right)\left(x_{\delta}\right)-\varphi\left(t_{\delta}, x_{\delta}\right) \\
& =\inf _{\alpha \in \Gamma_{t_{\delta}, t_{\delta}+\gamma \delta}^{S P}} \sup _{b \in \mathcal{B}_{t_{\delta}, t_{\delta}+\gamma \delta}}\left[\int_{t_{\delta}}^{t_{\delta}+\gamma \delta} l\left(s, x_{\delta}(s), \alpha[b](s), b(s)\right) d s\right. \\
& \left.\quad+F_{t_{\delta}+\gamma \delta, t_{\delta}+\delta}^{P} \varphi\left(t_{\delta}+\delta, \cdot\right)\left(x_{\delta}\left(t_{\delta}+\gamma \delta\right)\right)\right]-\varphi\left(t_{\delta}, x_{\delta}\right) \\
& =\inf _{\alpha \in \Gamma_{t_{\delta}, t_{\delta}+\gamma \delta}^{S P}} \sup _{b \in \mathcal{B}_{t_{\delta}, t_{\delta}+\gamma \delta}}\left[\int_{t_{\delta}}^{t_{\delta}+\gamma \delta} l\left(s, x_{\delta}(s), \alpha[b](s), b(s)\right) d s+\varphi\left(t_{\delta}+\gamma \delta, x_{\delta}\left(t_{\delta}+\gamma \delta\right)\right)\right. \\
& \left.\quad+F_{t_{\delta}+\gamma \delta, t_{\delta}+\delta}^{P} \varphi\left(t_{\delta}+\delta, \cdot\right)\left(x_{\delta}\left(t_{\delta}+\gamma \delta\right)\right)-\varphi\left(t_{\delta}+\gamma \delta, x_{\delta}\left(t_{\delta}+\gamma \delta\right)\right)\right]-\varphi\left(t_{\delta}, x_{\delta}\right)
\end{aligned}
$$

where $x_{\delta}(\cdot)$ is solution of (1.1) with initial condition $x_{\delta}\left(t_{\delta}\right)=x_{\delta}$ and controls $\alpha[b], b$. By continuity on initial condition (2.7) and Proposition 2.6,

$$
\begin{aligned}
F_{t_{\delta}+\gamma \delta, t_{\delta}+\delta}^{P} \varphi\left(t_{\delta}+\delta, \cdot\right)\left(x_{\delta}\left(t_{\delta}+\gamma \delta\right)\right) & -\varphi\left(t_{\delta}+\gamma \delta, x_{\delta}\left(t_{\delta}+\gamma \delta\right)\right) \\
& =(1-\gamma)\left[\frac{\partial \varphi}{\partial t}(t, x)+\underline{H}(t, x, \nabla \varphi(t, x))\right] \delta+o(\delta), \delta \rightarrow 0+
\end{aligned}
$$

where $o(\delta)$ is uniform on $\alpha, b$. Thus, from (4.2) and Proposition 2.5, we have

$$
\begin{aligned}
& F_{t_{\delta}, t_{\delta}+\delta}^{(\gamma)} \varphi\left(t_{\delta}+\delta, \cdot\right)\left(x_{\delta}\right)-\varphi\left(t_{\delta}, x_{\delta}\right) \\
& =\inf _{\alpha \in \Gamma_{t_{\delta}, t_{\delta}+\gamma \delta}^{S P}} \sup _{b \in \mathcal{B}_{t_{\delta}, t_{\delta}+\gamma \delta}}\left[\int_{t_{\delta}}^{t_{\delta}+\gamma \delta} l\left(s, x_{\delta}(s), \alpha[b](s), b(s)\right) d s+\varphi\left(t_{\delta}+\gamma \delta, x_{\delta}\left(t_{\delta}+\gamma \delta\right)\right)\right] \\
& \quad+(1-\gamma)\left[\frac{\partial \varphi}{\partial t}(t, x)+\underline{H}(t, x, \nabla \varphi(t, x))\right] \delta-\varphi\left(t_{\delta}, x_{\delta}\right)+o(\delta) \\
& \quad+\gamma\left[\frac{\partial \varphi}{\partial t}(t, x)+\bar{H}(t, x, \nabla \varphi(t, x))\right] \delta+(1-\gamma)\left[\frac{\partial \varphi}{\partial t}(t, x)+\underline{H}(t, x, \nabla \varphi(t, x))\right] \delta+o(\delta) \\
& \left.=\frac{\partial \varphi}{\partial t}(t, x)+H^{(\gamma)}(t, x, \nabla \varphi(t, x))\right] \delta+o(\delta)
\end{aligned}
$$

This completes the proof of the present proposition.

For given partition $\pi: 0=t_{0}<t_{1}<\cdots<t_{k}=T$, define $V_{\pi}^{(\gamma)}:[0, T] \times \mathbb{R}^{N} \rightarrow \mathbb{R}$ recursively backward in time:

$$
\begin{aligned}
V_{\pi}^{(\gamma)}(T, x) & =\Phi(x), x \in \mathbb{R}^{N} \\
V_{\pi}^{(\gamma)}(t, x) & =F_{t, t_{i+1}} V_{\pi}^{(\gamma)}\left(t_{i+1}, \cdot\right)(x), x \in \mathbb{R}^{N} \text { if } t_{i} \leq t<t_{i+1} .
\end{aligned}
$$

We consider the asymptotics of $V_{\pi}^{(\gamma)}$ as $\|\pi\| \equiv \max _{0 \leq i \leq k}\left|t_{i+1}-t_{i}\right| \rightarrow 0$. As we noted in Section 2, under (A.1)-(A.3), it is proved that $V^{P}, V^{S P}$ are bounded and Lipschitz 
continuous in $[0, T] \times \mathbb{R}^{N}$. Similarly, we can see that $V_{\pi}^{(\gamma)}$ is also bounded and Lipschitz continuous. Moreover, it is not difficult to show that $L^{\infty}$-bound and Lipschitz constant of $V_{\pi}^{(\gamma)}$ on $[0, T] \times \mathbb{R}^{N}$ are independent of $\pi$. Therefore, by Ascoli-Arzelà's theorem, there exist a subsequence $\left\{V_{\pi^{(n)}}^{(\gamma)}\right\}_{n=1}^{\infty}$ and a bounded Lipschitz continuous function $V^{(\gamma)}$ such that

$$
V_{\pi^{(n)}}^{(\gamma)}(t, x) \rightarrow V^{(\gamma)}(t, x) \text { as }\left\|\pi^{(n)}\right\| \rightarrow 0 \text { uniformly on each compact set in }[0, T] \times \mathbb{R}^{N} .
$$

Indeed, we can prove $V^{(\gamma)}$ is a viscosity solution of (3.3) with $H=H^{(\gamma)}$, i.e. we have the following result:

Theorem 4.2. $V_{\pi}^{(\gamma)}$ converges uniformly on compact set in $[0, T] \times \mathbb{R}^{N}$ to the unique viscosity solution $V^{(\gamma)} \in B U C\left([0, T] \times \mathbb{R}^{N}\right)$ of Isaacs equation $(3.3)$ with $H=H^{(\gamma)}$ :

$$
\left\{\begin{array}{c}
\frac{\partial V^{(\gamma)}}{\partial t}(t, x)+H^{(\gamma)}\left(t, x, \nabla V^{(\gamma)}(t, x)\right)=0, \quad(t, x) \in(0, T) \times \mathbb{R}^{N} \\
V^{(\gamma)}(T, x)=\Phi(x), x \in \mathbb{R}^{N} .
\end{array}\right.
$$

Proof. Note that under (A.1)-(A.3), we can apply uniqueness or comparison results of viscosity solution in $B U C\left([0, T] \times \mathbb{R}^{N}\right)$ to (4.6) (cf. [7], [1]). So, we prove that if $V_{\pi}^{(\gamma)}$ converges to $V^{(\gamma)}$ uniformly on compact sets, then $V^{(\gamma)}$ is a viscosity solution of (4.6).

Let $(t, x) \in(0, T) \times \mathbb{R}^{N}$ be a local maximum point of $V^{(\gamma)}-\varphi$ for sufficiently smooth function $\varphi$ on $(0, T) \times \mathbb{R}^{N}$. Specifically, we take $[t-r, t+r] \times \bar{B}_{r}(x) \subset(0, T) \times \mathbb{R}^{N}$ such that

$$
V^{(\gamma)}(s, y)-\varphi(s, y) \leq V^{(\gamma)}(t, x)-\varphi(t, x), \quad(s, y) \in[t-r, t+r] \times \bar{B}_{r}(x) .
$$

We may suppose that $(t, x)$ is a strictly local maximum point without loss of generality. For each partition $\pi: 0=t_{0}^{\pi}<t_{1}^{\pi}<\cdots \leq t_{k}^{\pi}=T$, take maximum point $\left(t_{\pi}, x_{\pi}\right)$ of $V_{\pi}^{(\gamma)}-\varphi$ in $[t-r, t+r] \times \bar{B}_{r}(x)$. Since $(t, x)$ is a strict maximum point and $V_{\pi}^{(\gamma)}$ converges to $V^{(\gamma)}$ uniformly on compact sets, we see that

$$
\left(t_{\pi}, x_{\pi}\right) \rightarrow(t, x) \text { as }\|\pi\| \rightarrow 0 .
$$

Therefore, we have for sufficiently small $\|\pi\|$,

$$
\left(t_{\pi}, x_{\pi}\right) \in[t-r / 2, t+r / 2] \times \bar{B}_{r / 2}(x) .
$$

By definition of $V_{\pi}^{(\gamma)}$,

$$
V_{\pi}^{(\gamma)}\left(t_{\pi}, x_{\pi}\right)=F_{t_{\pi}, t_{i+1}^{\pi}}^{(\gamma)} V_{\pi}^{(\gamma)}\left(t_{i+1}^{\pi}, \cdot\right)\left(x_{\pi}\right) \text { if } t_{i}^{\pi} \leq t_{\pi}<t_{i+1}^{\pi} .
$$

Indeed, (4.9) reads

$$
\begin{aligned}
\inf _{\alpha \in \Gamma_{t_{\pi}, t_{\pi}+\gamma \delta_{\pi}}^{S P}} \sup _{b \in \mathcal{B}_{t_{\pi}, t_{\pi}+\gamma \delta_{\pi}}}\left[\int_{t_{\pi}}^{t_{\pi}+\gamma \delta_{\pi}}\right. & l(s, x(s) \alpha[b](s), b(s)) d s \\
& \left.+F_{t_{\pi}+\gamma \delta_{\pi}, t_{i+1}^{\pi}}^{P} V_{\pi}^{(\gamma)}\left(t_{i+1}^{\pi}, \cdot\right)\left(x\left(t_{\pi}+\gamma \delta_{\pi}\right)\right)\right]-V_{\pi}^{(\gamma)}\left(t_{\pi}, x_{\pi}\right)=0
\end{aligned}
$$


where we set $\delta_{\pi} \equiv t_{i+1}^{\pi}-t_{\pi}$ Then, by (2.7), (4.8) and the assumption $V_{\pi}^{(\gamma)}$ converges to $V^{(\gamma)}$ uniformly on compact sets, we have for sufficiently small $\|\pi\|$,

$$
\begin{aligned}
F_{t_{\pi}+\gamma \delta_{\pi}, t_{i+1}^{\pi}}^{P} V_{\pi}^{(\gamma)}\left(t_{i+1}^{\pi}, \cdot\right)\left(x\left(t_{\pi}+\gamma \delta_{\pi}\right)\right)- & V_{\pi}^{(\gamma)}\left(t_{\pi}, x_{\pi}\right) \\
& \leq F_{t_{\pi}+\gamma \delta_{\pi}, t_{i+1}^{\pi}}^{P} \varphi\left(t_{i+1}^{\pi}, \cdot\right)\left(x\left(t_{\pi}+\gamma \delta_{\pi}\right)\right)-\varphi\left(t_{\pi}, x_{\pi}\right),
\end{aligned}
$$

which is uniform on $\alpha$ and $b$. Thus, we obtain

$$
0 \leq F_{t_{\pi}, t_{i+1}^{\pi}} \varphi\left(t_{i+1}^{\pi}, \cdot\right)\left(x_{\pi}\right)-\varphi\left(t_{\pi}, x_{\pi}\right)
$$

Hence, by using Proposition 4.1, we can see that

$$
\frac{\partial \varphi}{\partial t}(t, x)+H^{(\gamma)}(t, x, \nabla \varphi(t, x))=\lim _{\|\pi\| \rightarrow 0} \frac{F_{t_{\pi}, t_{i+1}^{\pi}} \varphi\left(t_{i+1}^{\pi}, \cdot\right)\left(x_{\pi}\right)-\varphi\left(t_{\pi}, x_{\pi}\right)}{t_{i+1}^{\pi}-t_{\pi}} \geq 0 .
$$

Therefore, $V^{(\gamma)}$ is a viscosity subsolution of (4.6).

The proof of supersolution is proved in a similar way.

\section{References}

[1] M. Bardi and I. Capuzzo-Dolcetta, Optimal Control and Viscosity Solutions of Hamilton-Jacobi-Bellman Equations, Birkhäuser, Boston, 1997.

[2] T. Basar and P. Bernhard, $H^{\infty}$-optimal control and related minimax design problems, 2nd ed., Birkhäuser, Boston, 1995.

[3] R.J. Elliott and N.J. Kalton, The existence of value in differential games, Mem. Amer. Math. Soc. 126, 1972.

[4] L.C. Evans and P.E. Souganidis, Differential games and representation formulas of Hamilton-Jacobi-Isaacs equations, Indiana Univ. Math. J. 33(5) (1984), 773-797.

[5] W.H. Fleming, Max-plus stochastic control, Lecture Notes on Control and Info. Sci. 280 (ed. B.Pasik Duncan) (2002), 111-119.

[6] W.H. Fleming, Max-plus stochastic Processes, preprint.

[7] W.H. Fleming and H.M. Soner, Controlled Markov Processes and Viscosity Solutions, Springer-Verlag, 1991.

[8] E. Roxin, The axiomatic approach in differential games, J. Optim. Theory Appl. 3 (1969), 153-163.

[9] P.E. Souganidis, Approximation schemes for viscosity solutions of Hamilton-Jacobi equations, J. Differential Eq. 59 (1985), 1-43. 
[10] P.E. Souganidis, Max-min representations and product formulas for the viscosity solutions of Hamilton-Jacobi equations with applications to differential games, Nonlinear Analysis, Theory, Methods \& Appl. 9(3) (1985), 217-257.

[11] P.P. Varaiya, The existence of solutions to a differential game, SIAM J. Control Optim., 5 (1967), 153-162. 